\title{
Conformational and vibrational study of cis-diamminedichloropalladium(II) $\dagger$
}

\author{
Sónia M. Fiuza, ${ }^{a}$ Ana M. Amado, ${ }^{a}$ Hélio F. Dos Santos, ${ }^{b c}$ Maria P. M. Marques ${ }^{a}$ \\ and Luis A. E. Batista de Carvalho ${ }^{a}$
}

Received 22nd June 2010, Accepted 25th August 2010

DOI: $10.1039 / \mathrm{c0cp00957a}$

\begin{abstract}
A conformational and vibrational analysis of $c$ is-diamminedichloropalladium(II) (cDDPd) is reported. Several theoretical methods (from Hartree-Fock to Møller-Plesset and Density Functional Theory) combined with different all-electron basis-sets are evaluated, in view of determining the best suited strategy for accurately representing this molecule. This choice is based on the best compromise between accuracy and computational requirements. Different scaling models were tested for obtaining the best scaling schemes of the vibrational modes to be used in this type of inorganic systems. The structural parameters and vibrational results predicted by the calculations are compared with the corresponding experimental data, namely X-ray structure and vibrational spectra. Finally, a complete assignment of the cDDPd vibrational spectra is presented.
\end{abstract}

\section{Introduction}

After the discovery of cisplatin's (cis-diamminedichloroplatinum(II), cDDP) anticancer properties, ${ }^{1}$ the interest in metal-based chemotherapeutic agents increased noticeably. Over the years, a large number of palladium(II) compounds with cytotoxic activity emerged, ${ }^{2}$ challenging the initial concepts that complexes comprising this metal centre would be biologically inactive and solely useful for their recognized catalytic properties. This early belief was due to the reported lack of activity of the parent compound cis-diamminedichloropalladium(II) (cDDPd) ${ }^{3}$ and to the fact that, although platinum(II) and palladium(II) share similar chemical properties, the latter is much more labile. ${ }^{4}$ As a result, the strategies for the development of biologically active $\mathrm{Pd}(\mathrm{II})$ complexes are presently oriented towards the management of this inherent lability, achieved by changing the nature of the ligands and/or preventing cis/trans isomerisation, for instance.

In order to allow the rational design of new and more efficient $\mathrm{Pd}(\mathrm{II})$ antineoplastic agents it is essential to better understand their chemical-physical properties and the structureactivity relationships (SAR's) ruling their interaction with the biological agent. Quantum mechanical calculations are a powerful tool for attaining the former objective, especially when combined with experimental data such as vibrational spectroscopy results. Although some theoretical studies on Pd(II) complexes have been previously reported, ${ }^{5}$ nor a

${ }^{a}$ Química-Física Molecular, Departamento de Química, FCTUC,

Universidade de Coimbra, P-3004-535 Coimbra, Portugal.

E-mail: sonia.mfiuza@gmail.com; Fax: +351239826541;

Tel: +351239826541

${ }^{b}$ NEQC: Núcleo de Estudos em Química Computacional,

Departamento de Química, Universidade Federal de Juiz de Fora, 36.036-330, Juiz de Fora-MG, Brazil

${ }^{c}$ NuBio-JF: Núcleo de Bioinformática de Juiz de Fora, Universidade Federal de Juiz de Fora, 36.036-330,

Juiz de Fora - MG, Brazil

$\dagger$ Electronic supplementary information (ESI) available: cDDPd experimental IR spectrum and different scaling schemes applied to the calculated wavenumbers. See DOI: $10.1039 / \mathrm{c} 0 \mathrm{cp} 00957 \mathrm{a}$ complete conformational analysis or a clear prediction of the corresponding minima in the potential energy surface (PES) have been obtained.

Currently, there is a wide variety of computational software, theoretical approaches and basis-sets that can be used to study the target compounds. This renders the choice of the calculation method far from straightforward and leads to abundant results, obtained at a variety of levels of theory. The aim of this work is to test a number of basis-sets in order to determine the best ones for attaining a "parameterization" of the calculation of palladium(II) complexes.

This study considers different theoretical methods such as HF (Hartree-Fock), DFT (density functional theory) and MP2 (second-order Møller-Plesset perturbation theory) combined to small and large basis-sets, with double and triple-zeta split valence, in view of achieving the best relationship between accuracy and computational effort ratio for cDDPd. This is particularly important as the complexity of the systems increases, especially when polynuclear Pd-complexes are considered.

The accuracy of the calculated results is verified by comparing the calculated values with the experimental ones, namely X-ray structural data $^{5 e}$ and the vibrational spectra.

The present study not only contributes to evaluate and complete previous studies on cDDPd vibrational analysis, but it also delivers important information for the vibrational study of cDDPd-related systems, an emerging class of new anticancer drugs.

\section{Experimental section}

Computational details

All the calculations were performed on a personal computer, using the Gaussian 03W (G03W) package and a Linux release (G03 D.01) ${ }^{6}$ installed in the clusters of the Núcleo de Estudos em Química Computacional in the Universidade Federal de Juiz de Fora, Brasil (NEQ C-UFJF).

Geometries were fully optimized by the Berny algorithm, using redundant internal coordinates, within symmetry 
constraints. The optimization convergence criteria for the cut-offs of forces and step sizes considered for the selfconsistent field (SCF) calculations were: 0.000015 hartree bohr $^{-1}$ for maximum force, 0.000010 hartree $\mathrm{bohr}^{-1}$ for root-meansquare force, $0.000060 \mathrm{bohr}$ for maximum displacement and 0.000040 bohr for root-mean-square displacement (keyword $o p t=t i g h t)$. In all cases, vibrational frequency calculations were performed, at the same level of theory, in order to verify that the geometries are a real minimum in the potential energy surface (no imaginary eigenvalues) as well as to quantify the zero-point vibrational energy (zpve) and thermal corrections.

For the non-metal atoms, different all-electron basis-sets (AE) were tested. Two different valence splits were considereddouble and triple-split valence - as well as the effect of the introduction of polarization and diffuse functions with Pople's ${ }^{7}$ and Dunning's correlation consistent basis-sets (Table 1). In all cases the relativistic pseudopotentials developed by Hay and $\mathrm{Wadt}^{8}{ }^{8}$ in a double-zeta splitting scheme, were used, as implemented in G03 (keyword LANL2DZ), to describe the palladium atom.

In order to evaluate the effect of electron correlation (known to be particularly meaningful in systems containing transition metal atoms ${ }^{9}$ ), different theoretical methods were considered. Hence, the HF methodology was evaluated with Pople's basissets (Dunning's correlation-consistent basis-sets were developed to be applied with correlated methods) while MP2 frozen core (FC) calculations, along with two different hydrid DFT protocols, were assessed with both Dunning's and Pople's AE basis-sets. The DFT protocols tested were: (i) the widely used B3LYP, which includes a mixture of HF and DFT exchange terms and the gradient-corrected functionals of Lee, Yang and Parr, as proposed and parameterized by Becke, ${ }^{10}$ and (ii) mPW1PW, which comprises a modified version of the exchange term of Perdew-Wang and the Perdew-Wang 91 correlation functional. ${ }^{11}$ For the DFT method, the two-electron integrals calculations were evaluated with a pruned grid of 75 radial shells and 302 angular points per shell (G03W keyword grid $=75302$, specifying a FineGrid), since a pruned grid of 99 radial shells and 590 angular points per shell (G03W keyword grid $=99590$, defining an UltraFineGrid) was not found to affect the results (data not shown) as verified in a previous study. ${ }^{12}$

The MP2(full)/AUG-cc-pVTZ level of theory was used for the preliminary determination of the possible conformations of cDDPd. This was the most extensive level of theory applied and the closest attempt to the calculation method limit, and thus the probable closest approximation to the experimental value. Since it was previously shown for other Pd(II) systems that the MP2 methodology yields similar results to the ones obtained with MP4, ${ }^{13}$ the latter was not considered. The values obtained at the MP2(full)/AUG-cc-pVTZ level of theory were used as an additional reference for assessing the quality of the results yielded by the other methods (MP2(FC), HF, B3LYP and $\mathrm{mPW} 1 \mathrm{PW})$. The different theoretical approaches are collected in Table 1. As stated, one of the aims of the present work is to find a theoretical method that yields the best prediction of the experimental data available for cDDPd, at the lowest computational cost. This was achieved by comparing the calculated values of both structural parameters and vibrational frequencies with the corresponding experimental values, similarly to a previous reported work ${ }^{12}$ (for further details on the accuracy evaluation procedure please check ref. 12).

\section{Synthesis}

The synthesis of cDDPd was performed according to Kirik et al. ${ }^{14}$ Briefly, cis-[$\left[\mathrm{Pd}\left(\mathrm{NH}_{3}\right)_{2} \mathrm{Cl}_{2}\right]$ was prepared using $\left[\mathrm{Pd}\left(\mathrm{NH}_{3}\right)_{2} \mathrm{C}_{2} \mathrm{O}_{4}\right]$ as the starting material. A $15 \%$ excess of $\mathrm{HCl}$ was mixed vigorously with $\left[\mathrm{Pd}\left(\mathrm{NH}_{3}\right)_{2} \mathrm{C}_{2} \mathrm{O}_{4}\right]$ for $2 \mathrm{~min}$, at room temperature. The suspension colour changed from pale to deep yellow. The compound was filtered, washed with ethanol and let to dry. Powder X-ray was performed and compared to the data reported by Kirik et al. ${ }^{14}$ confirming the presence of the cis isomer.

\section{Vibrational details}

Room-temperature Fourier transform Raman (FT-Raman) spectra were recorded on a RFS-100 Bruker FT-spectrometer,

Table 1 Compilation of the different methodologies and basis-sets (implemented in Gaussian 03 package) used for the conformational and vibrational study of cDDPd

\begin{tabular}{|c|c|c|c|c|}
\hline \multirow[b]{2}{*}{ Methodology } & \multicolumn{2}{|l|}{$\underline{\text { Basis sets }}$} & \multirow[b]{2}{*}{ Type } & \multirow[b]{2}{*}{ Atoms } \\
\hline & Double-zeta & Triple-zeta & & \\
\hline $\begin{array}{l}\text { HF } \\
\text { MP2 (full and frozen core) } \\
\text { DFT (mPW1PW and B3LYP) }\end{array}$ & $\begin{array}{l}\text { 6-31G } \\
6-31 \mathrm{G}(\mathrm{d}) \\
6-31 \mathrm{G}(\mathrm{d}, \mathrm{p}) \\
6-31+\mathrm{G} \\
6-31++\mathrm{G} \\
6-31+\mathrm{G}(\mathrm{d}) \\
6-31+\mathrm{G}(\mathrm{d}, \mathrm{p}) \\
6-31++\mathrm{G}(\mathrm{d}) \\
6-31++\mathrm{G}(\mathrm{d}, \mathrm{p}) \\
6-31+\mathrm{G}(2 \mathrm{df}) \\
6-31+\mathrm{G}(2 \mathrm{df}, \mathrm{p}) \\
6-31+\mathrm{G}(2 \mathrm{df}, 2 \mathrm{p}) \\
6-31++\mathrm{G}(2 \mathrm{df}, 2 \mathrm{pd}) \\
- \\
\text { cc-pVDZ } \\
\text { AUG-cc-pVDZ } \\
\text { LANL2DZ }\end{array}$ & $\begin{array}{l}6-311 G \\
6-311 G(d) \\
6-311 G(d, p) \\
6-311+G \\
6-311++G \\
6-311+G(d) \\
6-311+G(d, p) \\
6-311++G(d) \\
6-311++G(d, p) \\
6-311+G(2 d f) \\
6-311+G(2 d f, p) \\
6-311+G(2 d f, 2 p) \\
6-311++G(2 d f, 2 p d) \\
6-311++G(3 d f, 3 p d) \\
\text { cc-pVTZ } \\
\text { AUG-cc-pVTZ }\end{array}$ & $\begin{array}{l}\text { Dunning } \\
\text { ECP }\end{array}$ & $\mathrm{H}, \mathrm{N}, \mathrm{Cl}$ \\
\hline
\end{tabular}


using an Nd:YAG laser with an excitation wavelength of $1064 \mathrm{~nm}$. Each spectrum is the average of three repeated measurements of 150 scans, at a $2 \mathrm{~cm}^{-1}$ resolution.

The Fourier transform infrared (FTIR) spectra were recorded at room-temperature, over the $400-4000 \mathrm{~cm}^{-1}$ region, on a Mattson 7000 FTIR spectrometer, using a globar source, a deuterated triglycine sulfate (DTGS) detector and potassium bromide pellets. Each spectrum was composed of 32 scans, with a $2 \mathrm{~cm}^{-1}$ resolution and triangular apodization.

\section{Results and discussion}

Using the highest level of theory, MP2(full)/AUG-cc-pVTZ, and taking into consideration the free rotation of the amine groups, four geometries (Fig. 1) - two with $C_{2 \mathrm{v}}$ symmetry (cDDPd1 and cDDPd3), one $C_{\mathrm{s}}(\mathrm{cDDPd} 2)$ and one of $C_{2}$ (cDDPd0) symmetry - were found on the gas phase potential energy surface (PES) for cDDPd, and considered in the further calculations.

The goal of the present work was to perform a conformational, structural and vibrational study at lower theory levels and compare the results with both those obtained at the MP2(full)/AUG-cc-pVTZ level of theory and the experimental data available for the system. This study was carried out with a variety of basis-sets, combined with different methodologies, in view of evaluating the effect of including electron correlation and enlarging the basis-set with either diffuse or different sets of polarization functions.

\section{Conformational analysis}

The conformational analysis presently carried out aimed at determining the number of conformations and energy minima obtained for each level of theory. The energy differences between conformers were compared for three distinct situations: (i) without correction (raw energies); (ii) using the zero-point vibrational energy (zpve) correction; (iii) using the Gibbs free energy $(\Delta G)$ values for correction of the raw energies, where, in addition to zpve, thermal and entropic corrections are taken into account.
MP2 results. The conformational results obtained at the MP2(full)/AUG-cc-pVTZ level of theory are depicted in Fig. 2. This level of theory predicts cDDPd0, cDDPd 2 and cDDPd3 as stable conformers (minimum energy points on the PES) and cDDPd1 as a saddle point (Fig. 2A). The energy difference between conformers after $\Delta G$ correction renders cDDPd0 as the global minimum. However, these energy differences are very small, which might indicate that in the solid state the packing forces should be enough to stabilize any single conformer in the crystalline cell.

MP2 frozen core (FC) methodology does not predict the same conformers for the variety of basis-set tested, being highly dependent on the basis-set used (Fig. 2A). None of the considered levels mimetizes the results obtained at MP2(full)/AUG-cc-pVTZ, and only a few basis-sets are able to predict cDDPd0 geometry as a stationary point on the PES with MP2(FC) (namely, 6-31++G, 6-31+G*, 6-31++ $\mathrm{G}^{*}$, $6-31+\mathrm{G}^{* *}$ and $\left.6-31++\mathrm{G}^{* *}\right)$. The majority of these basis-sets identify cDDPd 1 as the single minimum conformer, in opposition to the prediction obtained with MP2(full)/AUG-cc-pVTZ level of theory.

The relative energies between stable conformers are presented in Fig. 2B. For each level of theory, the energies of the conformations are referred to cDDPd1 (cDDPd1's energy is subtracted to the energy of the other conformers since this is the most frequent conformer found for most methodologies). It can be observed that the energy minimum and the energy differences vary, not only between different levels of theory, but also with the type of correction performed, either with zpve or $\Delta G$. The zpve correction, however, yields strange results for $6-31++\mathrm{G}, 6-31++\mathrm{G}^{*}, 6-31+\mathrm{G}^{* *}$, 6-31++G**, 6-311G, 6-311G* and cc-pVTZ basis-sets, and even at the MP2(full)/AUG-cc-pVTZ level, with the saddle point (cDDPd1) being the lowest energy conformation. On the other hand, when applying $\Delta G$ correction, these problems are overcome, except for the MP2(FC) results with 6-311G and cc-pVTZ basis-sets, which lead to a cDDPd3 conformer with a higher Gibbs free energy than the cDDPd1 saddle point. It is opportune to mention that small energy differences are hard to predict, even at high levels of theory such as MP2(full)/AUG-cc-pVTZ, mainly when thermal corrections
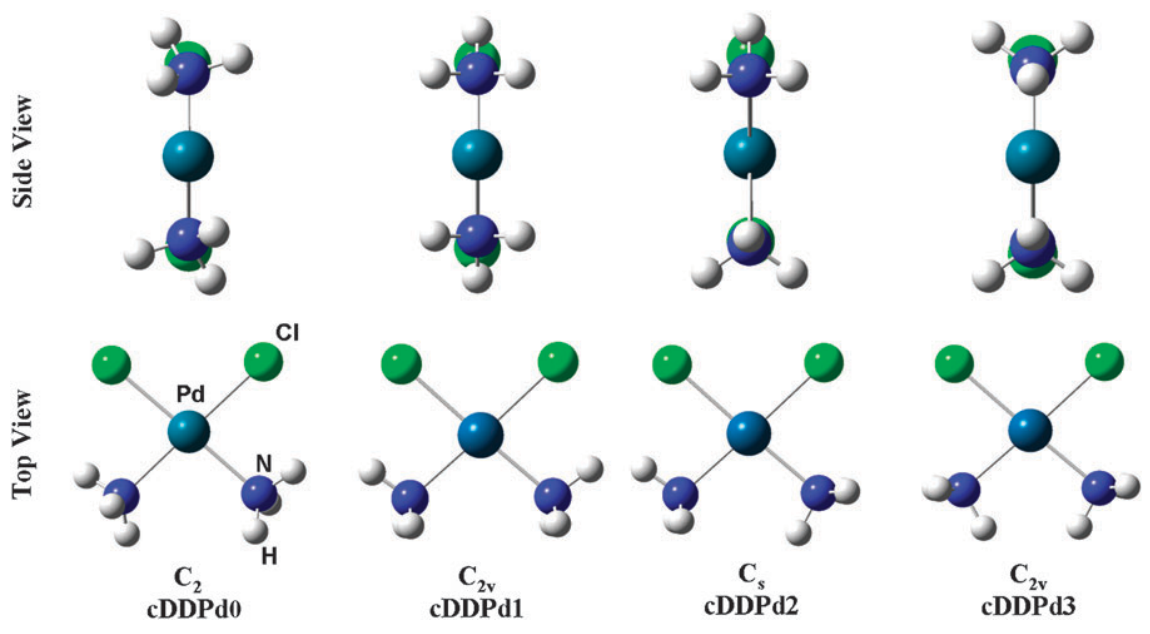

Fig. 1 Schematic representation of the different geometries obtained for cDDPd. 
A

First calculated vibrational frequency with MP2

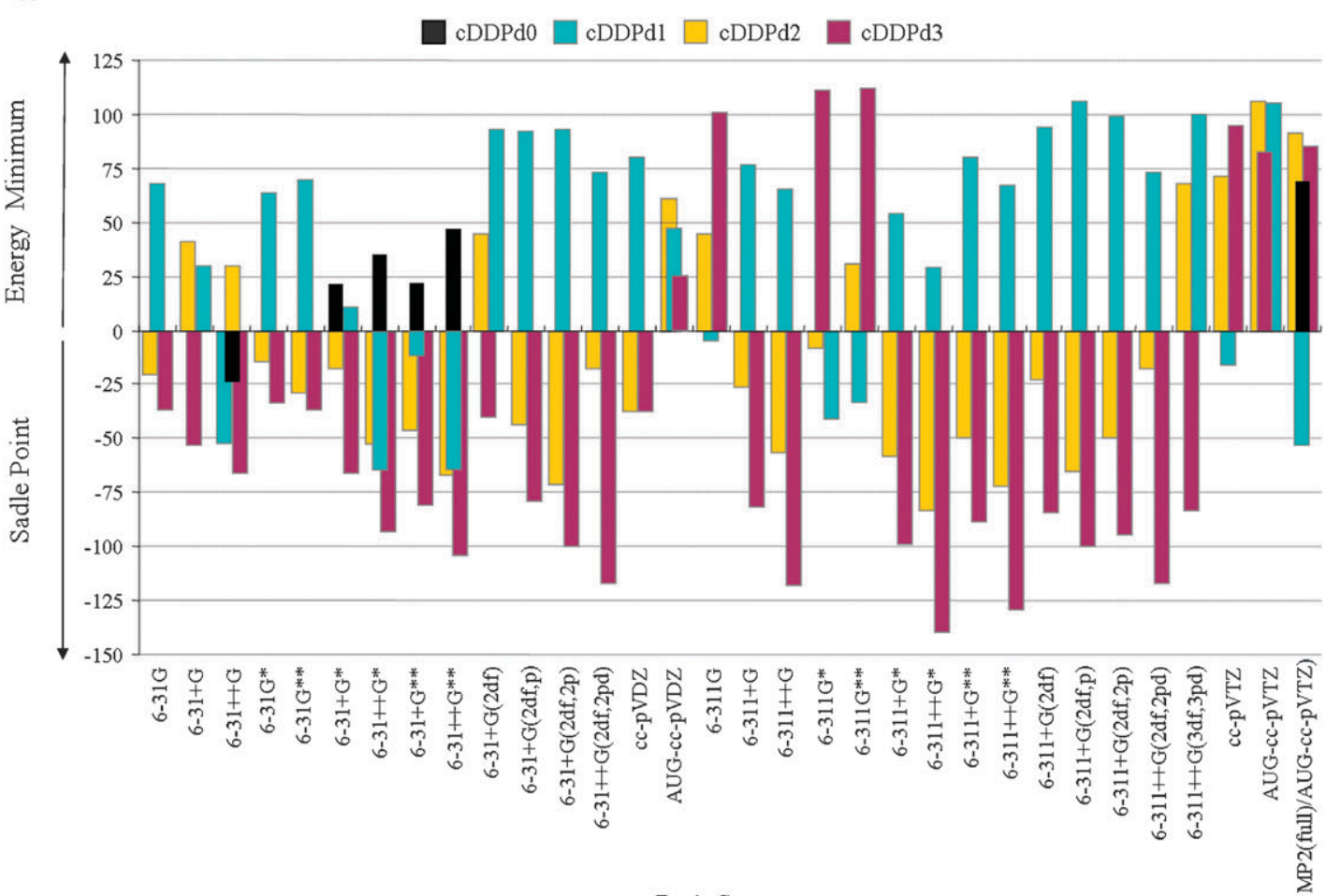

B

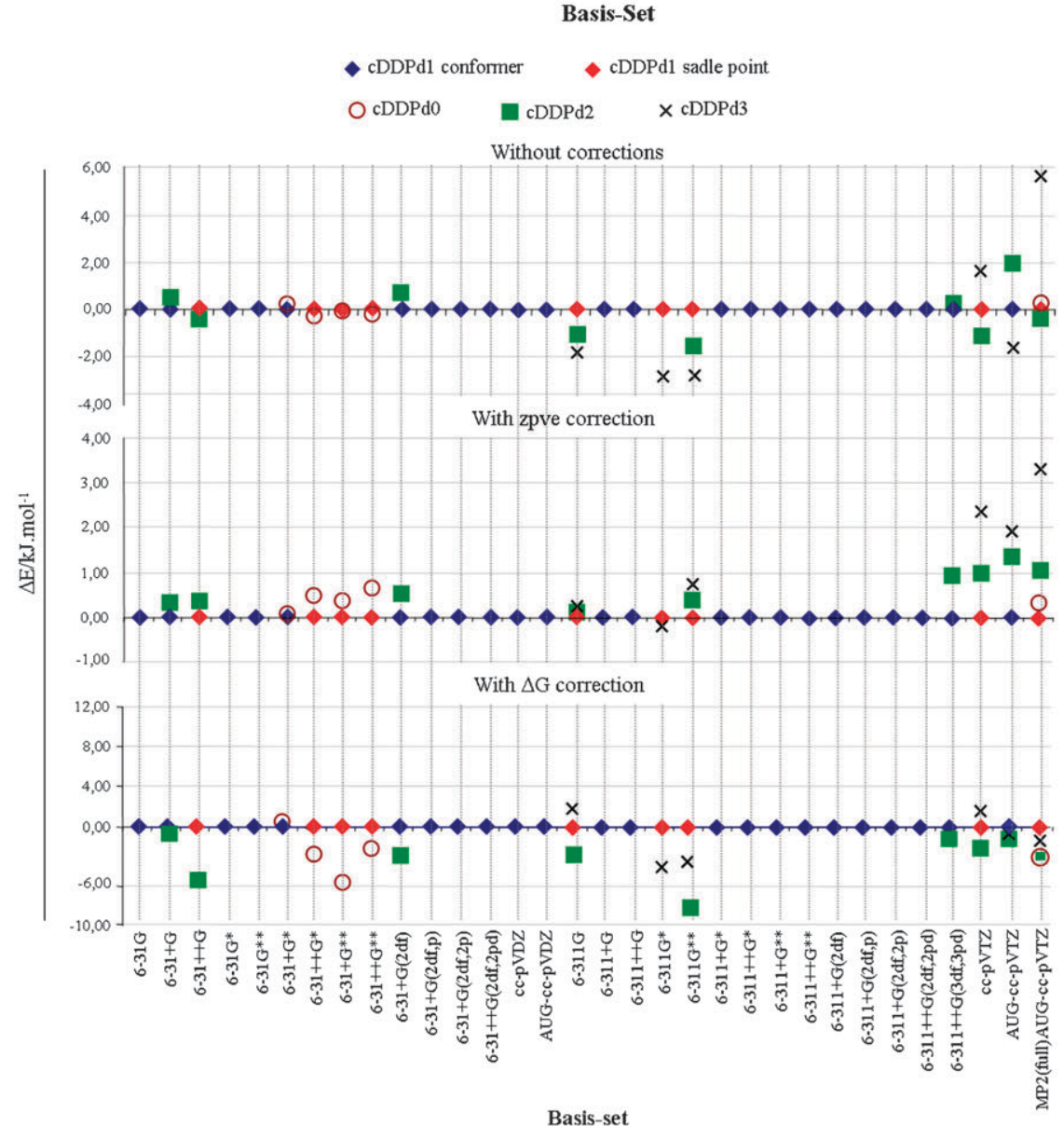

Fig. 2 Conformational analysis performed for cDDPd at MP2 methodology at the different theory levels. First vibrational transition $\left(\mathrm{cm}^{-1}\right)(\mathrm{A})$ and energy difference between conformers (B). 
are considered. ${ }^{15}$ Nevertheless, the effect of the theory level (including basis-set) on the local PES curvature must be considered as an important result for further studies.

HF results. The conformational results obtained with the non-correlated methodology $\mathrm{HF}$ are depicted in Fig. 3. The results obtained at the MP2(full)/AUG-cc-pVTZ are presented for reference. Firstly, it was found that the cDDPd0 geometry was predicted with one basis-set only and as a saddle point (6-31+G**; Fig. 3A). With the other basis-sets, this geometry converged to one of the other $C_{2 \mathrm{v}}$ configurations (either cDDPd1 or cDDPd3). Regarding the number of minimum energy conformers predicted (Fig. 3A), it can be observed that they range from two to three, depending on the basis-set used. The conformation cDDPd2 is, in all cases, an energy minimum, and when applying some triple-zeta quality basissets, only cDDPd1 is predicted as a saddle point.

The relative energy differences between conformers are presented in Fig. 3B. When considering the energy differences without any correction, one can observe that the relative energies are very small (below $2 \mathrm{~kJ} \mathrm{~mol}^{-1}$ ). After zpve correction, the results tend to become more uniform, with cDDPd 1 $\left(C_{2 \mathrm{v}}\right)$ being found as the lowest energy conformation with the majority of the basis-sets. The exceptions are the $6-311 \mathrm{G}^{*}$, 6-311+G* and $6-311++\mathrm{G}^{*}$ basis-sets, which predict cDDPd2 $\left(C_{2 \mathrm{v}}\right)$ as the lowest energy conformation. However, despite the tendency for homogeneity of the data, zpve correction leads to odd results yielding the saddle point as the lowest energy conformation $\left(6-31+\mathrm{G}^{* *}, 6-31++\mathrm{G}^{* *}, 6-311 \mathrm{G}, 6-311 \mathrm{G}^{* *}\right)$, as found in the raw energies when using $6-311++\mathrm{G}(2 \mathrm{df}, 2 \mathrm{pd})$ and $6-311++\mathrm{G}(3 \mathrm{df}, 3 \mathrm{pd})$ basis-sets. On the other hand, when applying $\Delta G$ correction, the results are quite different. Not only the energy differences between conformations increase, but the anomalous results are in most cases corrected. Interestingly, when comparing HF and MP2 data (Fig. 2B and 3B), cDDPd1 (or cDDPd0 for some basis-sets) is found to be the preferred geometry at MP2 while cDDPd2 is the global minimum at HF. This difference might be related to the poor $\mathrm{HF}$ description of weak hydrogen bonds $(\mathrm{N}-) \mathrm{H} \cdots \mathrm{Cl}$, which play an important role in the stabilization of both cDDPd0 and cDDPd 1 forms.

DFT results. Both DFT protocols presently used are by far more uniform than the previously described methods (HF and MP2) when predicting the conformers (Fig. 4A) and the lowest energy minimum (Fig. 4B). Within these DFT protocols, while B3LYP always yields cDDPd1 as the single conformer, the same result is obtained with all basis-sets except one (6-311G*) within the mPW1PW protocol. The exception is mPW1PW/6-311G* theory level which predicts two stable conformations - cDDPd1 and cDDPd2 (Fig. 4A). In any case, the DFT protocols were not able to predict the $\operatorname{cDDPd} 0\left(C_{2}\right)$ geometry obtained at the MP2(full)/AUG-cc-pVTZ level of theory. In fact, for every combination tested the cDDPd0 geometry always converged to cDDPd1. This could be partly due to the overweight attributed to the intramolecular $(\mathrm{N}-) \mathrm{H} \cdots \mathrm{Cl}$ interactions in this methodology that leads to the stabilization of the cDDPd1 geometry. Nevertheless, the non-correlated $\mathrm{HF}$ method also failed to predict $\mathrm{cDDPd} 0$ as well as cDDPd1 as the main geometries, therefore suggesting a strong dispersion contribution to the $(\mathrm{N}-) \mathrm{H} \cdots \mathrm{Cl}$ interaction in the cDDPd0 form. ${ }^{15}$

In sum, the number of predicted conformers, as well as their relative conformational energies, strongly depends on both the theoretical method and the AE basis-set used to describe the non-metal atoms. The type of basis-set affects both the number of conformers and the magnitude of the relative stability orders. From $6-311 \mathrm{G}$ to $6-311+\mathrm{G}(2 \mathrm{df})$, some unexpected results were found that may be due to the fact that the $6-311 \mathrm{G}$ standard basis-set and related ones are not of real triple-zeta quality and thus the results should be analyzed with care. ${ }^{16}$

The strong dependence of MP2 on the basis-set has been verified before ${ }^{17}$ and is an evidence of the sensitivity of these type of systems to electron correlation. Regarding the choice of the basis-set, it is reported that for MP2 methodology larger basis-sets lead to better overall results. ${ }^{18}$ At the DFT level, however, improving the basis-set quality to a very high extent is somewhat not very useful since the overall DFT error is considerably larger than the basis-set error. ${ }^{19}$ On the whole, independently of the basis-set considered, DFT calculations yield mainly only one conformer (cDDPd1, similarly to MP2 level with most of the basis-sets) and larger energy differences between optimized geometries, as compared to HF and MP2 approaches. This probably results from the different weight attributed by the distinct methods (DFT, HF and MP2) to the intramolecular $(\mathrm{N}-) \mathrm{H} \cdots \mathrm{Cl}$ interactions. This seems to be underestimated within HF formalism (thus favoring the cDDPd2 form, which has only one $(\mathrm{N}-) \mathrm{H} \cdots \mathrm{Cl}$ hydrogen bond) and overestimated with DFT protocols (favoring the cDDPd1 form displaying two $(\mathrm{N}-) \mathrm{H} \cdots \mathrm{Cl}$ close contacts). At the MP2 methodology in turn, the symmetric form cDDPd1 $\left(C_{2 \mathrm{v}}\right)$ is also found as the global minimum. A few exceptions occur, namely the results obtained at the highest level of theory considered, which predicted the $C_{2}$ structure (cDDPd0) as the most stable one. In these cases, the $C_{2 \mathrm{v}}$ conformer is characterized as a first order transition state, connecting two equivalent $C_{2}$ forms separated by a quite small energy difference (energy barrier) suggesting a fast equilibrium between them with the average structure found as $C_{2 \mathrm{v}}$.

\section{Structural analysis}

The optimized structural parameters obtained at the different levels of theory (Fig. 5-7) were gathered for cDDPd1, the predominant conformer obtained for most of the DFT and MP2 levels applied. The corresponding MP2(full)/AUG-ccpVTZ calculated values for the $C_{2}$ geometry are also included, for comparison.

Pd-Cl bond. The calculated and experimental $\mathrm{Pd}-\mathrm{Cl}$ bond lengths are depicted in Fig. 5, for the different methodologies and basis-set combinations presently tested. It can be observed that the non-correlated methodology HF predicts the worse values for this structural parameter. Interestingly, mPW1PW performs similarly to MP2, with the majority of the basissets. In fact, mPW1PW performs better than B3LYP for this structural parameter with all basis-sets, with an average difference of about $0.026 \mathrm{pm}$. In general, all the methodologies 

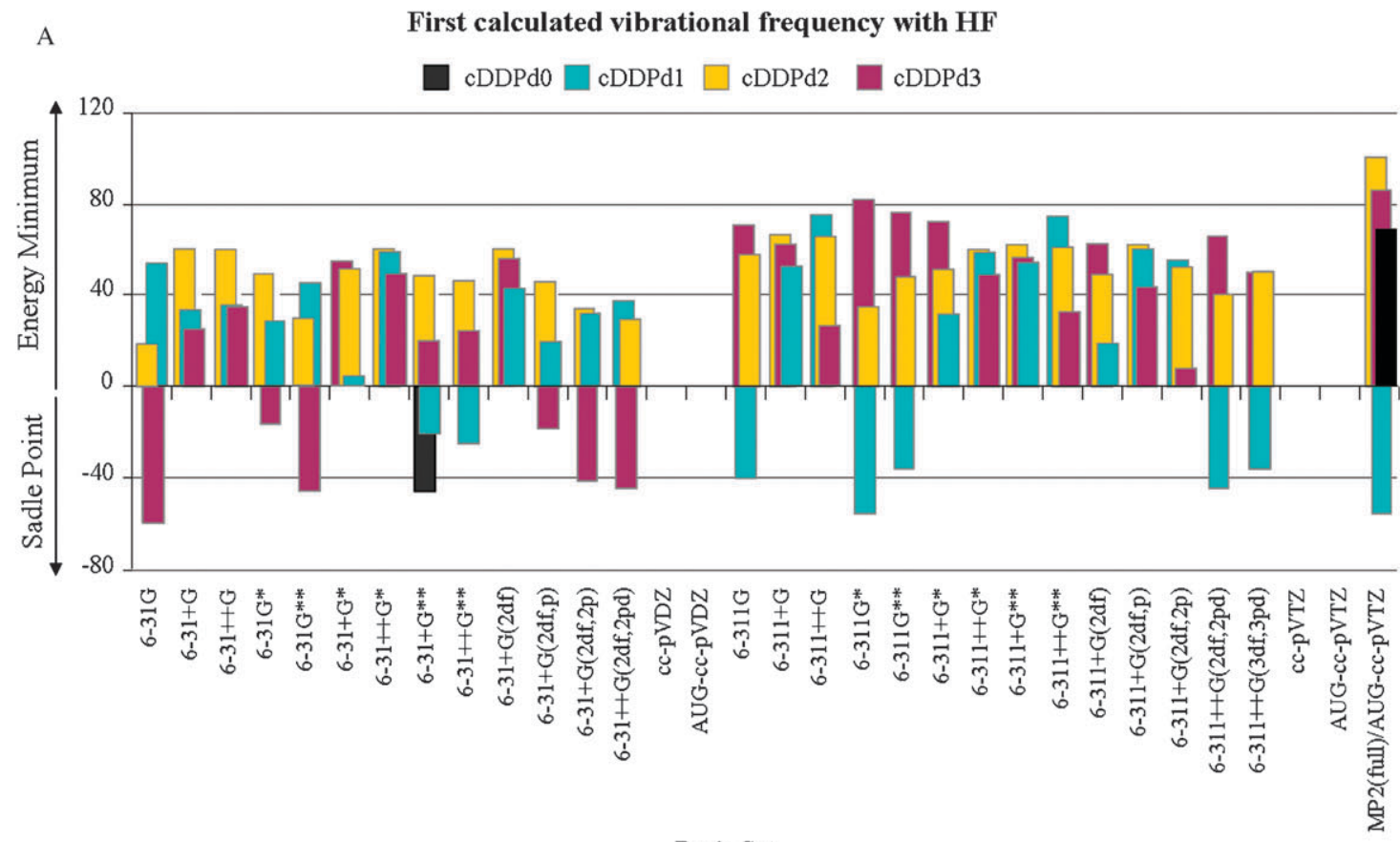

B

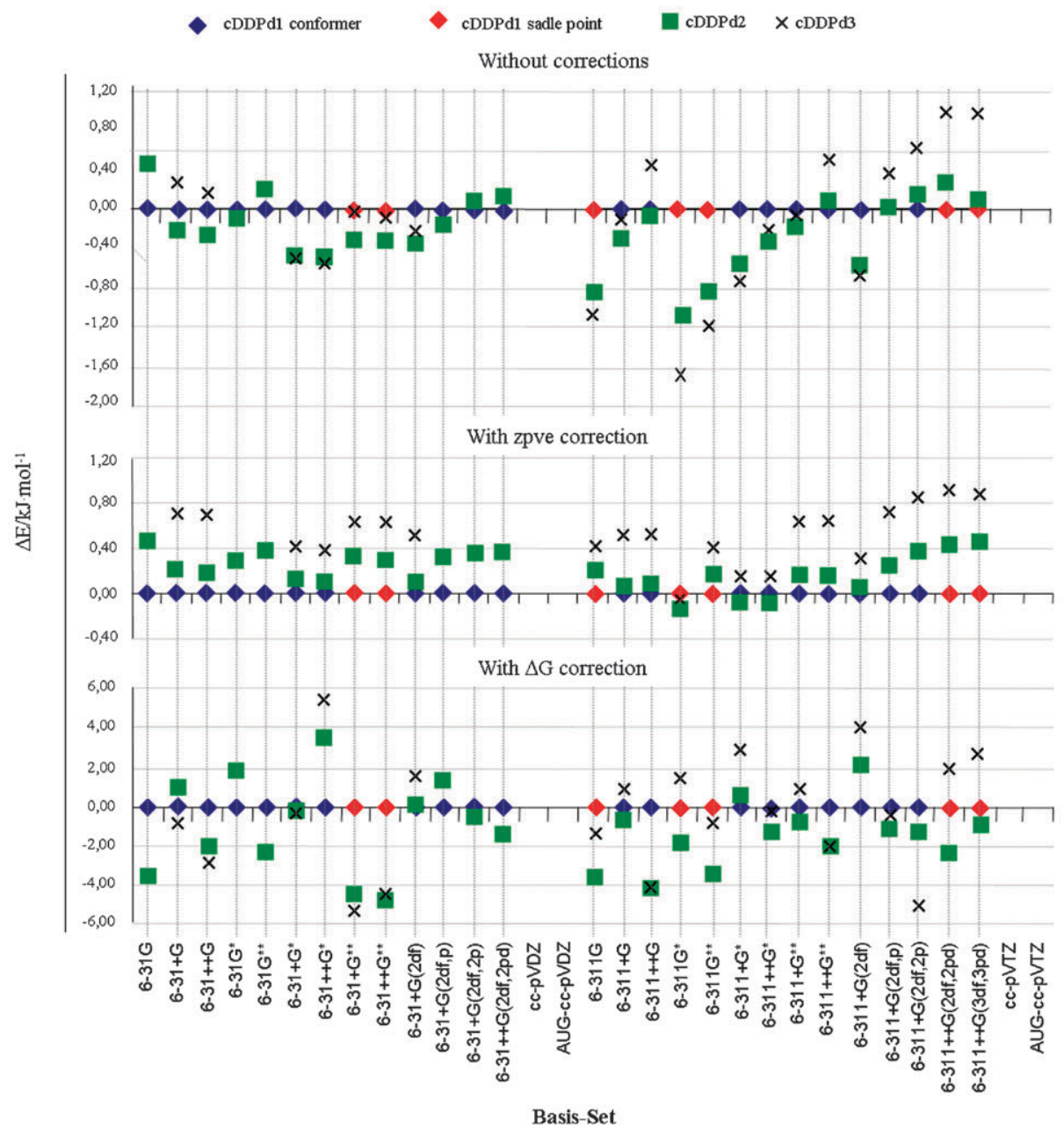

Fig. 3 Conformational analysis performed for cDDPd at HF methodology at the different theory levels. First vibrational transition ( $\left.\mathrm{cm}^{-1}\right)(\mathrm{A})$ and energy difference between conformers (B). 


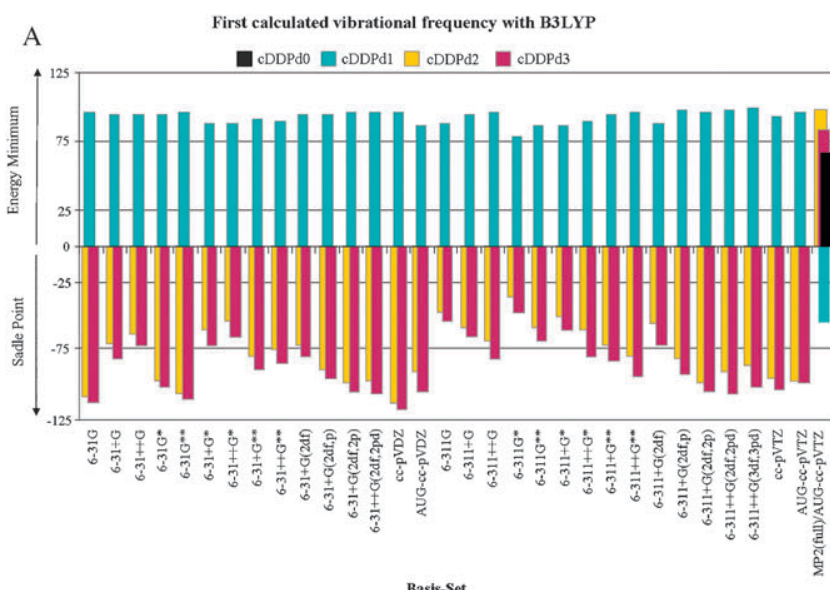

Basis-Set

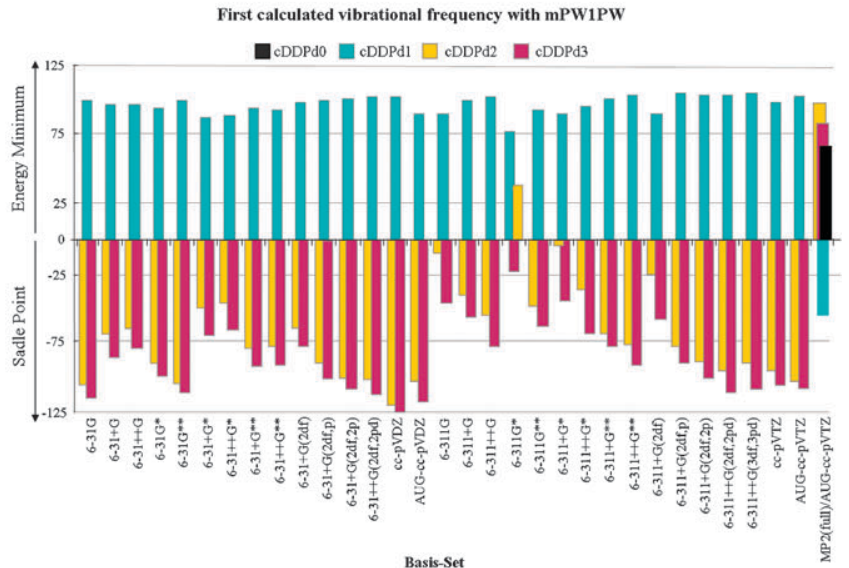

Basis-Set

cDDPd2

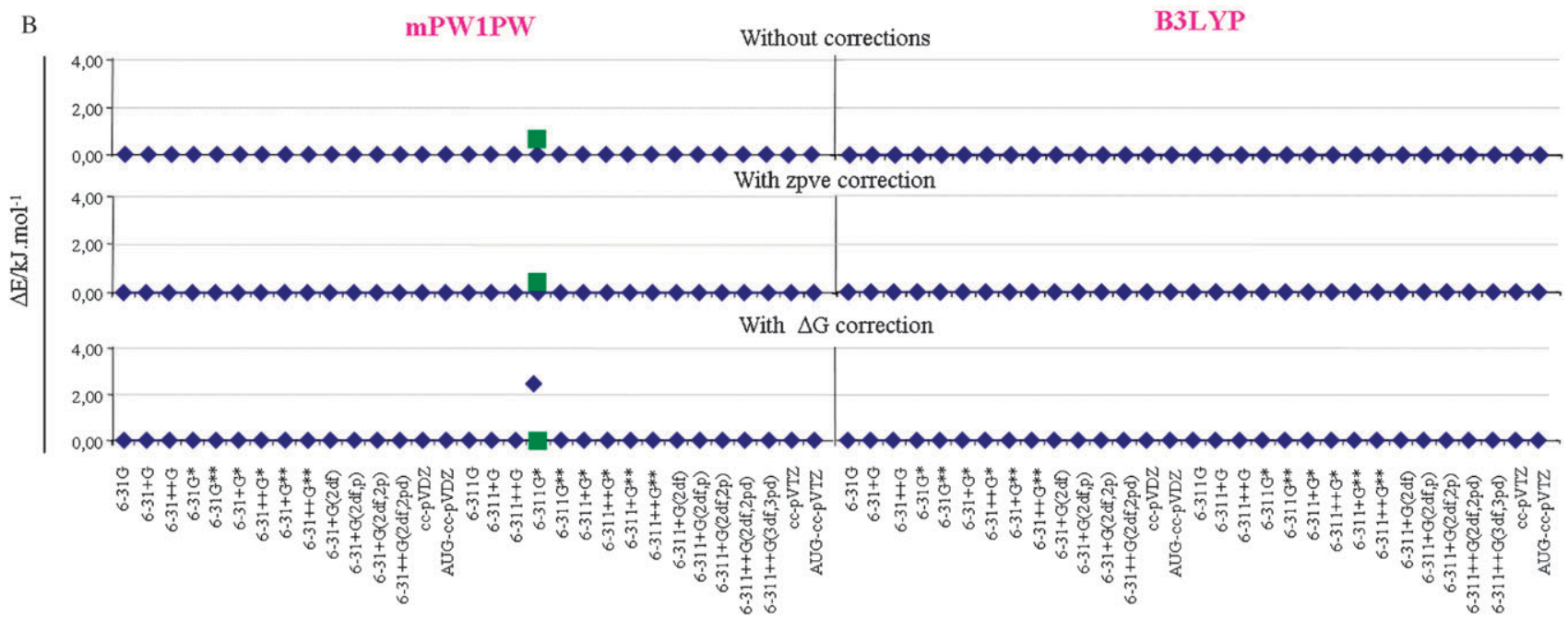

Basis-Set

Fig. 4 Conformational analysis performed for cDDPd at DFT methodology (B3LYP and mPW1PW) at the different theory levels. First vibrational transition $\left(\mathrm{cm}^{-1}\right)(\mathrm{A})$ and energy difference between conformers (B).

overestimate the $\mathrm{Pd}-\mathrm{Cl}$ bond length. The only exceptions are observed with the MP2 methodology coupled to the more demanding AUG-cc-pVTZ basis-sets.

The results obtained at the different methodologies present the same curve pattern and a similar response to the variation of the basis-set. As a consequence, the following analysis on the introduction of polarization and diffuse functions is carried out in general for all the methodologies tested. The introduction of polarization functions at the non-hydrogen atoms was found to cause a drastic reduction of the $\mathrm{Pd}-\mathrm{Cl}$ bond length towards the direction of the experimental value for the Pople's basis-sets. As expected, the introduction of a d-polarization function at the $\mathrm{Cl}$-atom is extremely important to describe the $\mathrm{Pd}-\mathrm{Cl}$ bond, since chloride bearing a fully occupied $p$ orbital is highly polarizable. These improvements can be verified in several situations: $6-31 \mathrm{G} \rightarrow 6-31 \mathrm{G}^{*} ; 6-31+\mathrm{G} \rightarrow 6-31+\mathrm{G}^{*}$; $6-31++\mathrm{G} \rightarrow 6-31++\mathrm{G}^{*} ; 6-311 \mathrm{G} \rightarrow 6-311 \mathrm{G}^{*} ; 6-311+\mathrm{G} \rightarrow$ $6-311+\mathrm{G}^{*} ; 6-311++\mathrm{G} \rightarrow 6-311++\mathrm{G}^{*}$. It is also worth noting the important role played by the second set of $\mathrm{d}$ polarization functions, augmented by the second-order polarization functions f. As shown in Fig. 5, the best results are achieved with basis-sets including $2 \mathrm{df}$ sets of polarization functions (double and triple-zeta quality), regardless of the theory level. This may be related to the better basis-set balance in the contracted schemes such as $6-31+\mathrm{G}(2 \mathrm{df})$ and $6-311+\mathrm{G}(2 \mathrm{df})$, which follow the standard rule according to which the number of functions of a given type should at most be one less than the type with one lower angular momentum. ${ }^{20}$

The introduction of a polarization function at the hydrogen atom has a negligible effect on the $\mathrm{Pd}-\mathrm{Cl}$ bond length, although one might have expected at least a slight effect on this bond due to a putative $(\mathrm{N}-) \mathrm{H} \cdots \mathrm{Cl}$ intramolecular interaction.

Regarding the introduction of diffuse functions on the double-zeta split valence basis-sets, either on the non-hydrogen atoms alone or at all atoms $(6-31 \mathrm{G} \rightarrow 6-31+\mathrm{G}$ and $6-31++\mathrm{G}$; $6-31 \mathrm{G}^{*} \rightarrow 6-31+\mathrm{G}^{*}$ and $6-31++\mathrm{G}^{*} ; 6-31 \mathrm{G}^{* *} \rightarrow 6-31+\mathrm{G}^{* *}$ and $6-31++G^{* *}$ and cc-pVDZ $\rightarrow$ AUG-cc-pVDZ), it was found that the bond length variation is very slight, with the MP2 methodology suffering the largest change ( $c a .0 .010 \mathrm{pm})$. When considering the triple-zeta valence basis-sets, the results 


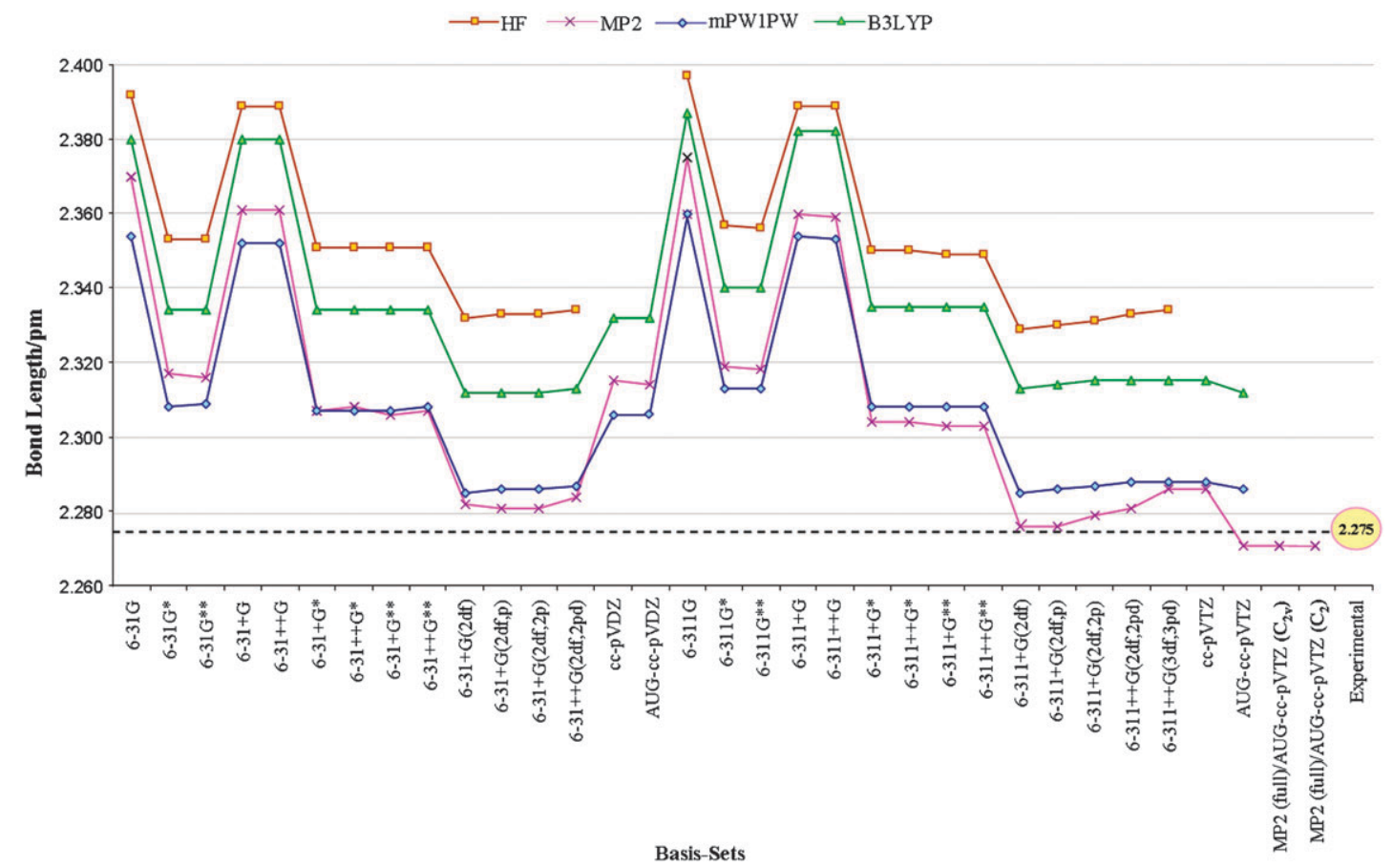

Fig. 5 Calculated values for the $\mathrm{Pd}-\mathrm{Cl}$ bond length considering different theory levels.

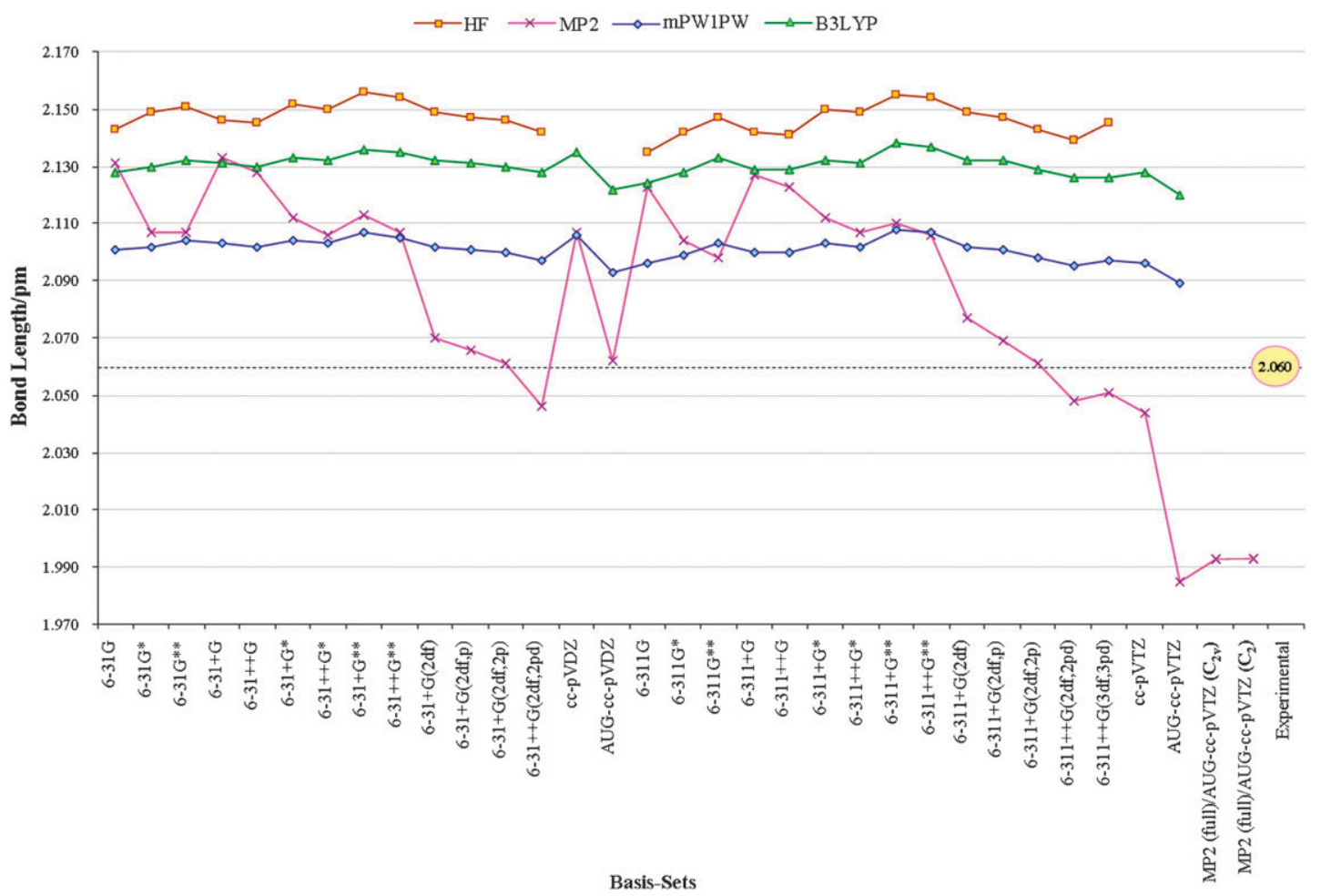

Fig. 6 Calculated values for the $\mathrm{Pd}-\mathrm{N}$ bond length considering different theory levels.

are more affected by the introduction of diffuse functions (ca. $0.015 \mathrm{pm})$.

Finally, considering the double-zeta split valence basis-sets, the best computational time $v s$. accuracy ration for the $\mathrm{Pd}-\mathrm{Cl}$ bond length value is obtained with the $6-31+\mathrm{G}(2 \mathrm{df})$ basis-set, within all four methods. Conversely, the MP2/6-311+G(2df) theory level yields the best accuracy-to-computational demands ratio when applying a triple-zeta split valence basis-set.

Pd-N bond. As for the $\mathrm{Pd}-\mathrm{Cl}$ parameter, the efficacy of the methods used follow the increasing efficacy trend $\mathrm{HF}<$ B3LYP $<$ mPW1PW, with MP2 being highly variable. Both 
A

$\longrightarrow \mathrm{HF} \rightarrow \mathrm{MP} 2 \longrightarrow \mathrm{mPW} 1 \mathrm{PW} \backsim \mathrm{B} 3 \mathrm{LYP}$

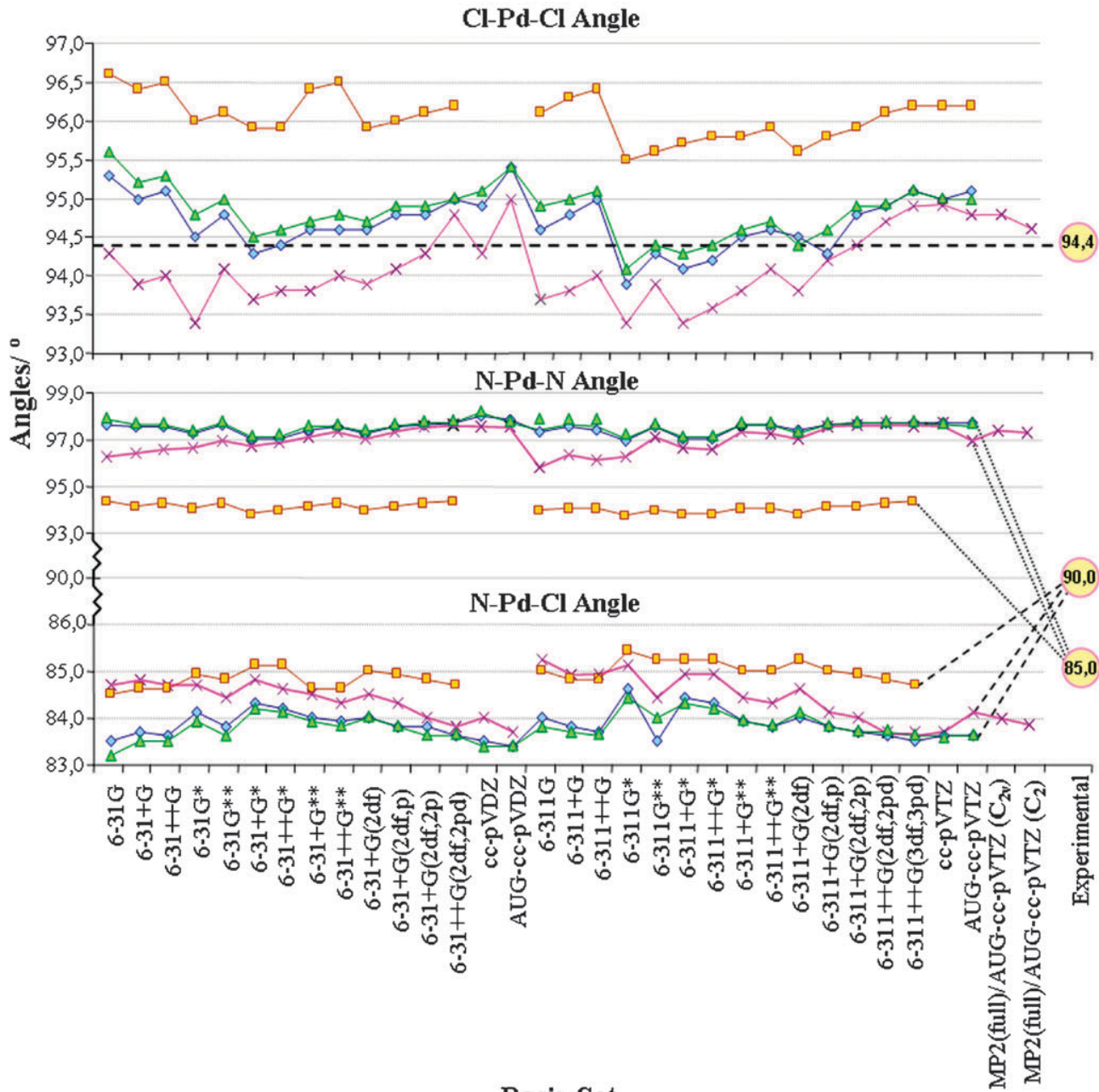

B

Basis-Set

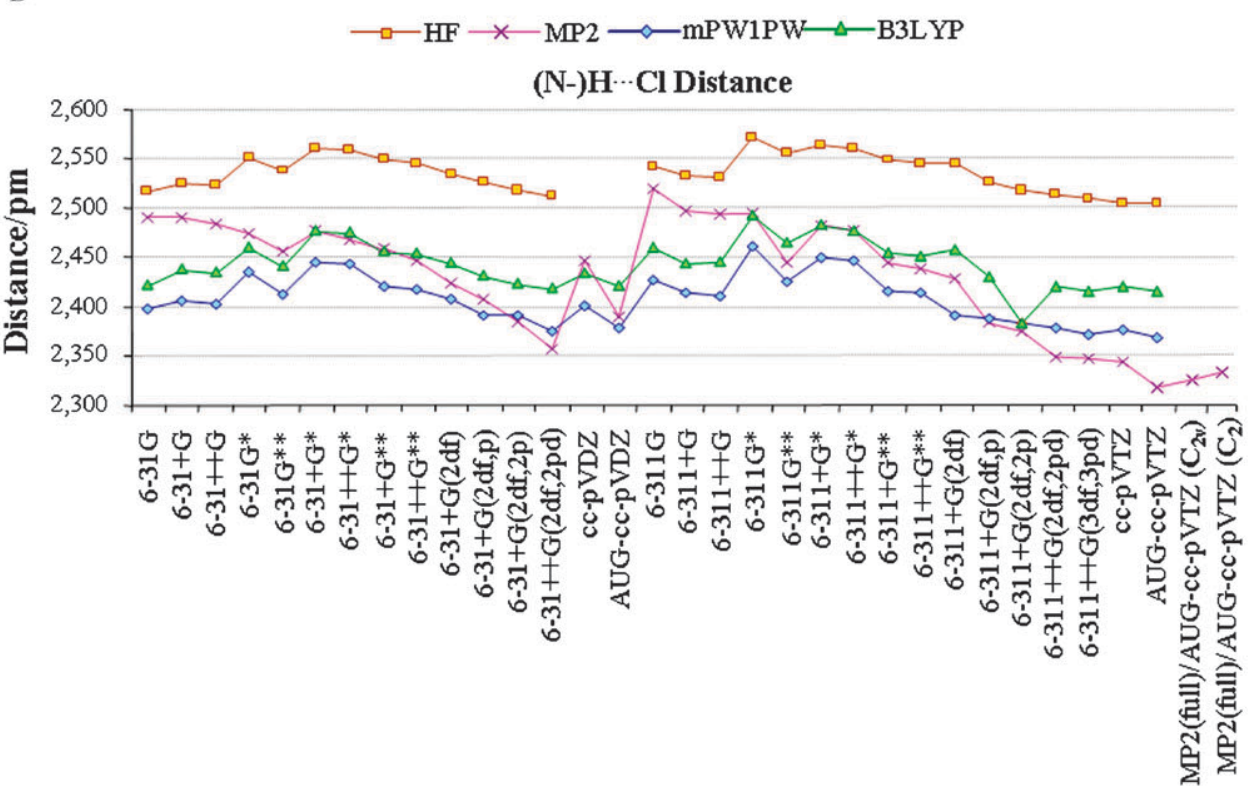

\section{Basis-Set}

Fig. 7 Calculated values for the angles $\mathrm{Cl}-\mathrm{Pd}-\mathrm{Cl}, \mathrm{N}-\mathrm{Pd}-\mathrm{N}$ and $\mathrm{N}-\mathrm{Pd}-\mathrm{Cl}(\mathrm{A})$ as well as for the intramolecular distance $(\mathrm{N}-) \mathrm{H} \cdots \mathrm{Cl}(\mathrm{B})$, considering different theory levels. 
$\mathrm{HF}$ and the DFT protocols were not as successful in reproducing this parameter as compared to MP2, leading to large errors and to low basis-set sensitivity (Fig. 6). The MP2 methodology, however, is able to reproduce this parameter quite well, mainly when coupled to either $6-31++\mathrm{G}(2 \mathrm{df}, 2 \mathrm{p})$ or 6-311++G(2df,2p) basis-sets.

The reason that this parameter is more difficult to reproduce may be related to the involvement of the $\mathrm{N}$-atom in intermolecular interactions in the solid state through the $\mathrm{NH}_{3}$ group by establishing $(\mathrm{N}-) \mathrm{H} \cdots \mathrm{Cl}$ close contacts with the neighboring molecules. This can also be inferred from the fact that, while the basis-sets with a poor description of the hydrogen atoms $(6-31+\mathrm{G}(2 \mathrm{df})$ and $6-31++\mathrm{G}(2 \mathrm{df}))$ were enough to describe the $\mathrm{Pd}-\mathrm{Cl}$ bond adequately, the basis-sets that better describe the $\mathrm{Pd}-\mathrm{N}$ bond are those with a better description of the $\mathrm{H}$-atoms. Similarly to the $\mathrm{Pd}-\mathrm{Cl}$ bond, the inclusion of $2 \mathrm{df}$ sets of polarization functions was found to improve significantly the $\mathrm{Pd}-\mathrm{N}$ bond length values (see Fig. 6).

Cl-Pd-CI angle. Overall, the HF and both DFT protocols used tend to overestimate the amplitude of the $\mathrm{Cl}-\mathrm{Pd}-\mathrm{Cl}$ angle (Fig. 7A). Once more, HF yields the worst estimates for this structural parameter, while B3LYP shows improved results, comparable to those obtained with the mPW1PW protocol. The MP2 methodology in turn, underestimates this value for most of the levels of theory tested. This parameter is therefore well described by all correlated methodologies and although an error-free value can be obtained with the triple-zeta basissets, good estimates are also obtained for less demanding theory levels such as $\mathrm{mPW} 1 \mathrm{PW} / 6-31 \mathrm{G}^{*}$.

$\mathbf{N}-\mathbf{P d}-\mathbf{N}$ angle. The $\mathrm{N}-\mathrm{Pd}-\mathrm{N}$ bond angle is widely overestimated by all methodologies presently studied, HF predicting the best values (Fig. 7A). This is most probably due to the poor description of the $(\mathrm{N}-) \mathrm{H} \cdots \mathrm{Cl}$ intramolecular interaction at this level. In fact, the correlated methods attribute a greater weight to the intramolecular distance $(\mathrm{N}-) \mathrm{H} \cdots \mathrm{Cl}$ and this deforms the $\mathrm{N}-\mathrm{Pd}-\mathrm{N}$ angle to a larger extent. Note that the $(\mathrm{N}-) \mathrm{H} \cdots \mathrm{Cl}$ becomes shorter (Fig. 7B), with the improvement of basis-sets and electron correlation. As can be seen, the $(\mathrm{N}-) \mathrm{H} \cdots \mathrm{Cl}$ distance shortening is more drastically observed as the description of the $\mathrm{H}$-atoms becomes increasingly better, with HF being the less and MP2 the most affected methods. B3LYP and MPW1PW describe this parameter equally well and MP2 yields a slightly better representation for some selected basis-sets.

N-Pd-Cl angle. As expected, due to the intramolecular $(\mathrm{N}-) \mathrm{H} \cdots \mathrm{Cl}$ interaction and the opening of the $\mathrm{N}-\mathrm{Pd}-\mathrm{N}$ angle, the $\mathrm{N}-\mathrm{Pd}-\mathrm{Cl}$ angle is underestimated. This situation is similar to the one occurring for the $\mathrm{N}-\mathrm{Pd}-\mathrm{N}$ angle, since it is affected by the same effect. Once again the methodologies that better describe this parameter are HF and MP2 with both DFT protocols having a similar performance.

The $(\mathrm{N}-) \mathrm{H} \cdots \mathrm{Cl}$ interaction, and possibly the repulsion between the vicinal $\mathrm{NH}_{3}$ groups are overwhelming effects that could probably be better studied through the calculation of dimeric structures (currently underway). Actually, these interactions in turn render the choice of the best methodology for the description of the structural parameters very difficult and should be overcome by these further studies.

\section{Vibrational analysis}

Usually, the calculated vibrational frequencies are overestimated relative to the experimental ones. This difference between the calculated harmonic frequencies and the experimental values is ascribed to the incomplete description of the electron-electron interaction and to the neglect of anharmonicity. ${ }^{21}$ Therefore, scaling the calculated data is a normal procedure in order to achieve a better accordance with the experimental wavenumbers. Although there is considerable information regarding scaling factors for organic compounds, ${ }^{22}$ the same is not valid for inorganic compounds.

Fig. 8 shows the experimental FT-Raman spectra recorded for solid cDDPd, in the $75-1800 \mathrm{~cm}^{-1}$ and $3000-3600 \mathrm{~cm}^{-1}$ spectral regions. The FTIR spectrum is presented in the ESI $\dagger$ (Fig. S1). Some regions are expanded in order to allow a better visualization of spectral details, otherwise undetectable. The wavenumbers presented are the ones used for the accuracy evaluation of the calculated vibrational frequencies (discussed below). The assignments of the main bands are comprised in Table 2. cDDPd displays 27 vibrational modes, all Ramanactive. Considering conformation cDDPd1 $\left(C_{2 \mathrm{v}}\right)$, these modes are distributed as $9 \mathbf{a}_{1}+5 \mathbf{a}_{2}+5 \mathbf{b}_{1}+8 \mathbf{b}_{2}$. The very-low frequency modes (below $150 \mathrm{~cm}^{-1}$ ), such as the $\mathrm{NH}_{3}$ torsions are not considered for scaling purposes, since they are extremely affected by the crystal network in the solid state.
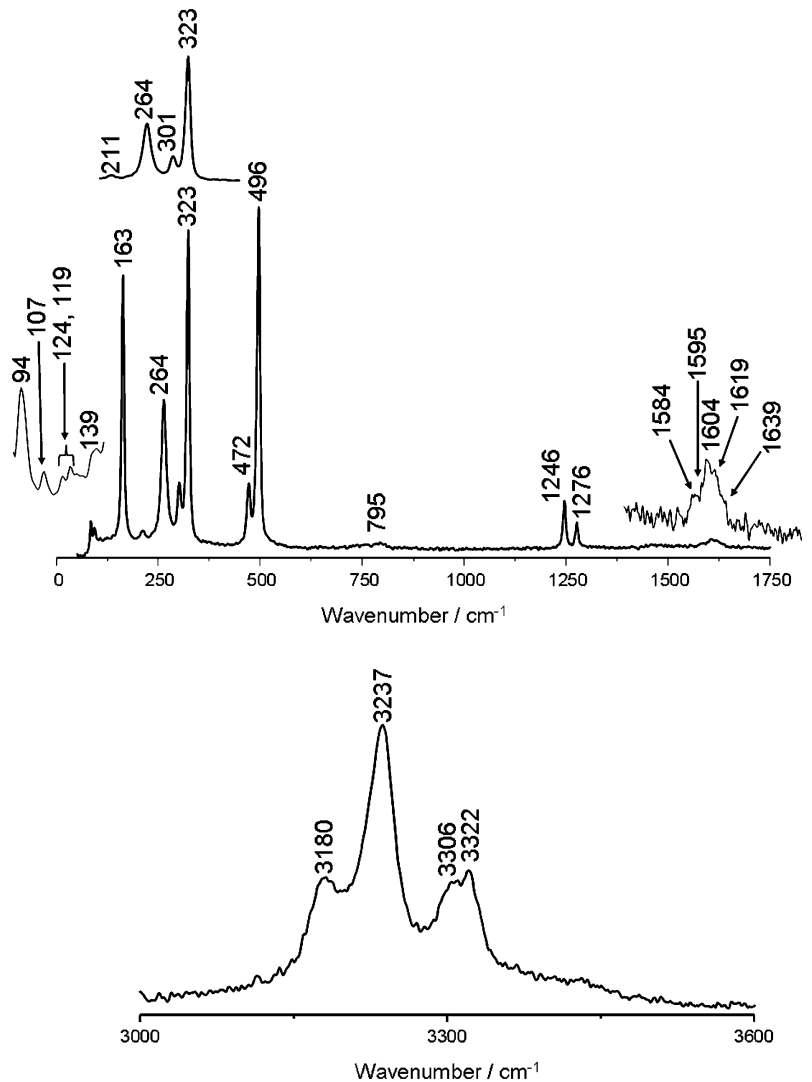

Fig. 8 Room-temperature FT-Raman spectra of solid cDDPd in the $50-1750$ and $3000-3600 \mathrm{~cm}^{-1}$ spectral regions. 
Table 2 Tentative assignment of cDDPd's vibrational modes (wavenumbers in $\mathrm{cm}^{-1}$ )

\begin{tabular}{|c|c|c|c|c|c|c|}
\hline \multirow[b]{2}{*}{ Assignments } & \multicolumn{4}{|c|}{ Calculated Raman active modes ${ }^{a}$} & \multicolumn{2}{|c|}{ Experimental } \\
\hline & $\mathrm{HF}$ & B3LYP & mPW1PW & $\mathrm{MP} 2(\mathrm{FC})$ & Raman & $\mathrm{IR}^{b}$ \\
\hline$\nu_{\text {as }} \mathrm{NH}_{3}$ & 3413 & 3465 & 3444 & 3408 & 3322 & \\
\hline$\nu_{\text {as }} \mathrm{NH}_{3}$ & 3387 & 3416 & 3389 & 3348 & 3306 & 3309 \\
\hline$\nu_{\mathrm{s}} \mathrm{NH}_{3}$ & 3288 & 3288 & 3248 & 3202 & 3237 & 3235 \\
\hline$\delta_{\text {as }} \mathrm{NH}_{3}$ & 1623 & 1617 & 1587 & 1562 & 1639 & 1636 \\
\hline$\delta_{\text {as }}^{\prime} \mathrm{NH}_{3}$ & 1614 & 1609 & 1578 & 1552 & 1619 & \\
\hline$\delta_{\text {as }} \mathrm{NH}_{3}$ & 1590 & 1579 & 1548 & 1506 & 1604 & 1611 \\
\hline$\delta^{\prime}{ }_{\text {as }} \mathrm{NH}_{3}$ & 1584 & 1574 & 1542 & 1498 & 1595 & 1591 \\
\hline$\delta_{\mathrm{s}} \mathrm{NH}_{3}$ & 1214 & 1329 & 1319 & 1309 & 1276 & 1269 \\
\hline$\delta_{\mathrm{s}}^{\prime} \mathrm{NH}_{3}$ & 1207 & 1321 & 1311 & 1295 & 1245 & 1248 \\
\hline$\rho \mathrm{NH}_{3}$ & 764 & 776 & 782 & 781 & 794 & 802 \\
\hline$\rho^{\prime} \mathrm{NH}_{3}$ & 720 & 731 & 740 & 738 & & 790 \\
\hline$\rho \mathrm{NH}_{3}$ & 709 & 731 & 738 & 731 & & 751 \\
\hline$\rho^{\prime} \mathrm{NH}_{3}$ & 682 & 699 & 707 & 684 & & 736 \\
\hline$\nu_{\mathrm{s}} \mathrm{Pd}-\mathrm{N}$ & 488 & 493 & 495 & 507 & 496 & \\
\hline$\nu_{\text {as }} \mathrm{Pd}-\mathrm{N}$ & 465 & 472 & 475 & 463 & 472 & 474 \\
\hline$\nu_{\mathrm{s}} \mathrm{Pd}-\mathrm{Cl}$ & 354 & 327 & 335 & 342 & 323 & \\
\hline$\nu_{\mathrm{as}} \mathrm{Pd}-\mathrm{Cl}$ & 318 & 301 & 311 & 320 & 301 & \\
\hline$\delta \mathrm{N}-\mathrm{Pd}-\mathrm{Cl}$ & 270 & 263 & 262 & 261 & 264 & \\
\hline$\delta \mathrm{N}-\mathrm{Pd}-\mathrm{N}$ & 229 & 203 & 208 & 223 & 211 & \\
\hline$\gamma \mathrm{N}-\mathrm{Pd}-\mathrm{Cl}$ & 169 & 173 & 177 & 183 & 163 & \\
\hline$\delta \mathrm{Cl}-\mathrm{Pd}-\mathrm{Cl}$ & 155 & 146 & 151 & 153 & & \\
\hline$\gamma^{\prime} \mathrm{N}-\mathrm{Pd}-\mathrm{Cl}$ & 110 & 126 & 132 & 133 & & \\
\hline$\tau \mathrm{NH}_{3}$ & 80 & 123 & 128 & 114 & & \\
\hline$\tau^{\prime} \mathrm{NH}_{3}$ & 50 & 100 & 104 & 100 & & \\
\hline
\end{tabular}

To determine the best scaling factors to be used at each theory level considered in the present work, different scaling schemes were tested, as previously reported. ${ }^{12,23}$ The results (scaling factors, vibrational modes comprised in each frequency set and rms values) for the distinct scaling schemes at the different theoretical levels are comprised in Tables S1-S5 $(\mathrm{ESI} \dagger)$. Only the scaling schemes yielding the lowest rms are herein presented (Table 3), which involves a three-factor frequency grouping scheme.

The first observation was that, for all theoretical levels, the best theoretical-to-experimental match is achieved with a set of three scaling factors. The use of a single factor (Tables S2 and $\mathrm{S} 3, \mathrm{ESI} \dagger)$ gives rise to very high $\mathrm{rms}$ values $(4.9 \% \leq \mathrm{rms} \leq$ $9.8 \%$ ), not leading to a substantial improvement relative to the error obtained without any scaling factor (Table S1, ESI $\dagger$; $5.5 \% \leq \mathrm{rms} \leq 11.1 \%)$. The use of two scaling factors (one above $\left(\lambda_{1}\right)$ and one below $400 \mathrm{~cm}^{-1}\left(\lambda_{2}\right)$, as suggested by Scott and Radom ${ }^{22 a}$ ) was shown not to improve the results (Tables S4 and S5, ESI $\dagger ; 4.9 \% \leq \mathrm{rms} \leq 12.0 \%$ ). Conversely, when three frequency sets are used, a considerable overall improvement of the results is achieved (Table $3 ; 1.5 \% \leq$ rms $\leq 5.8 \%$ ).

The results summarized in Table 3 suggest that the distribution of the vibrational frequencies into three groups is strongly dependent on the AE basis-set considered for describing the non-metal atoms. Moreover, independently of the basis-set, the wavenumbers corresponding to the amine stretching and deformation modes ( $\nu_{\mathrm{NH} 3}$ and $\delta_{\mathrm{NH} 3}$, respectively) are always overestimated by the calculations $\left(\lambda_{1}\right.$, Table 3$)$. On the other hand, all theoretical levels lead to a quite high underestimation of the $\mathrm{Pd}-\mathrm{N}$ stretching $\left(\nu_{\mathrm{Pd}-\mathrm{N}}\right)$ and $\mathrm{N}-\mathrm{Pd}-\mathrm{Cl}$ deformation $\left(\delta_{\mathrm{N}-\mathrm{Pd}-\mathrm{N}}\right)$ modes $\left(\lambda_{3}\right.$, Table 3$)$. The remaining vibrational modes, namely the four amine rocking modes $\left(\rho_{\mathrm{NH} 3}\right)$ and the skeletal $\mathrm{Cl}-\mathrm{Pd}-\mathrm{N}$ deformation $\left(\gamma_{\mathrm{Cl}-\mathrm{Pd}-\mathrm{Cl}}\right)$, are quite accurately predicted by all the methods tested $\left(\lambda_{2}\right.$, Table 3$)$.

It is interesting to note that for all scaling schemes mPW1PW is the methodology that performs the best, in opposition to what was previously observed for $\mathrm{CDDP}^{12}$ (B3LYP yielded the lowest rms values) and that for all methodologies the smaller basis-sets are the ones yielding the higher accuracy.

Finally, the overall profile of the Raman spectrum is analyzed regarding both frequency and intensity prediction. Only the cDDPd1 isomer was considered, its Raman spectrum having been computed with the expanded basisset 6-311++G(3df,3pd) at HF, DFT and MP2(FC) levels (see Table 2). The theoretical Raman intensities were derived from the Raman activities following the procedure proposed elsewhere. ${ }^{24}$ Fig. 9 presents the calculated spectra for cDDPd1 at MP2(FC)/6-311++G(3df,3pd) level, the band shapes having been plotted using a Lorentzian type function. ${ }^{25}$

The agreement between experimental-Fig. 8-and theoretical-Fig. 9-is fairly good, regardless of the method applied. The exception is the band calculated at $342 \mathrm{~cm}^{-1}$ (found experimentally at $323 \mathrm{~cm}^{-1}$ ) that is predicted to be quite intense compared to the one at $507 \mathrm{~cm}^{-1}$ (detected at $496 \mathrm{~cm}^{-1}$ ). It is important to note that when a comparison between experimental and theoretical spectra is made it should be taken into account that the calculated spectrum corresponds to a single molecule in an idealized perfect vacuum medium while the experimental spectrum is measured for a macroscopic sample that in this case exists in the solid state. Therefore, a perfect match between theoretical gas phase and the experimental spectra in the entire spectral range is not always possible and should not be expected. Thus, the spectroscopic analysis should concern the main Raman features of the spectrum (most intense and well resolved bands), which can be useful for a structural characterization and identification

\section{Conclusions}

In this work, a conformational study of cDDPd, the palladium analogue, to the well-known chemotherapeutic drug cisplatin was undertaken by quantum mechanical calculations within distinct methodologies-HF, DFT and MP2 - using several all-electron basis-sets to describe the non-metal atoms. The vibrational spectra of the molecule was calculated and compared with the experimental Raman, which allowed to build a set of scaling factors (for distinct groups of vibrational modes) yielding the best accordance between calculated and experimental results, and easily transferable to similar inorganic systems.

Depending on the theoretical approach, different energy minima were predicted for cDDPd, varying in the relative orientation of the $\mathrm{NH}_{3}$ ligands, with cDDPd1 being predicted as the lowest-energy geometry for the majority of the theory levels applied.

The assignment of the cDDPd experimental Raman spectra to be found in the literature are not up-to-date and are still 


\section{Acknowledgements}

The Portuguese Foundation for Science and Technology is acknowledged for financial support-Project PTDC/QUI/ 66701/2006 (co-financed by the European Community fund FEDER). HFDS would like to thank the Brazilian Agencies $\mathrm{CNPq}, \mathrm{CAPES}$ and FAPEMIG for supporting his laboratories. The authors further acknowledge Laboratório Associado CICECO (University of Aveiro, Portugal) for access to the FT-Raman and FT-IR spectrometers. SMF would like to thank Dr Leonid A. Solovyov (Institute of Chemistry and Chemical Technology, Krasnoyarsk 660049, Russia) for his kind help regarding the synthesis of $\mathrm{cDDPd}$.

\section{References}

1 B. Rosenberg, L. Vancamp and T. Krigas, Nature, 1965, 205, 698-699.

2 (a) A. Trevisan, C. Marzano, P. Cristofori, M. B. Venturini, L. Giovagnini and D. Fregona, Arch. Toxicol., 2002, 76, 262-268; (b) E. Budzisz, B. K. Keppler, G. Giester, M. Wozniczka, A. Kufelnicki and B. Nawrot, Eur. J. Inorg. Chem., 2004, 22, 4412-4419; (c) S. M. Fiuza, A. M. Amado, P. J. Oliveira, V. A. Sardão, L. A. E. Batista de Carvalho and M. P. M. Marques, Lett. Drug Des. Discovery, 2006, 3, 149-151.

3 M. J. Cleare and J. D. Hoeschele, Platinum Met. Rev., 1973, 17, $2-13$.

4 J. V. Burda, M. Zeizinger and J. Leszczynski, J. Chem. Phys., 2004, 120, 1253-1262.

5 (a) G. A. Hill, G. Forde, L. Gorb and J. Leszczynski, Int. J. Quantum Chem., 2002, 90, 1121-1128; (b) G. Yang, C. Jin, J. Hong, Z. Guo and L. Zhu, Spectrochim. Acta, Part A, 2004, 60, 3187-3195; (c) M. Zeizinger, J. V. Burda, J. Sponer, V. Kapsa and J. Leszczynski, J. Phys. Chem. A, 2001, 105, 8086-8092; (d) A. Milet and A. Dedieu, Theor. Chim. Acta, 1995, 92, 361-367; (e) S. D. Kirik, L. A. Solovyov, A. I. Blokhin and I. S. Yakimov, Acta Crystallogr., Sect. B: Struct. Sci., 2000, 56, 419-425.

6 M. J. Frisch, G. W. Trucks, H. B. Schlegel, G. E. Scuseria, M. A. Robb, J. R. Cheeseman, J. A. Montgomery Jr., T. Vreven, K. N. Kudin, J. C. Burant, J. M. Millam, S. S. Iyengar, J. Tomasi, V. Barone, B. Mennucci, M. Cossi, G. Scalmani, N. Rega, G. A. Petersson, H. Nakatsuji, M. Hada, M. Ehara, K. Toyota, R. Fukuda, J. Hasegawa, M. Ishida, T. Nakajima, Y. Honda, O. Kitao, H. Nakai, M. Klene, X. Li, J. E. Knox, H. P. Hratchian, J. B. Cross, V. Bakken, C. Adamo, J. Jaramillo, R. Gomperts, R. E. Stratmann, O. Yazyev, A. J. Austin, R. Cammi, C. Pomelli, J. W. Ochterski, P. Y. Ayala, K. Morokuma, G. A. Voth, P. Salvador, J. J. Dannenberg, V. G. Zakrzewski, S. Dapprich, A. D. Daniels, M. C. Strain, O. Farkas, D. K. Malick, A. D. Rabuck, K. Raghavachari, J. B. Foresman, J. V. Ortiz, Q. Cui, A. G. Baboul, S. Clifford, J. Cioslowski, B. B. Stefanov, G. Liu, A. Liashenko, P. Piskorz, I. Komaromi, R. L. Martin, D. J. Fox, T. Keith, M. A. Al-Laham, C. Y. Peng, A. Nanayakkara,
M. Challacombe, P. M. W. Gill, B. Johnson, W. Chen, M. W. Wong, C. Gonzalez and J. A. Pople, Gaussian 03, Revision D.01, Gaussian, Inc., Wallingford, CT, 2004.

7 M. J. Frisch, J. A. Pople and J. S. Binkley, J. Chem. Phys., 1984, 80, 3265-3269.

8 P. J. Hay and W. R. Wadt, J. Chem. Phys., 1985, 82, 299-310.

9 H. Chermette, Coord. Chem. Rev., 1998, 178-180, 699-721.

10 (a) A. D. Becke, Phys. Rev. A: At., Mol., Opt. Phys., 1988, 38, 3098-3100; (b) C. T. Lee, W. T. Yang and R. G. Parr, Phys. Rev. B: Condens. Matter, 1988, 37, 785-789.

11 (a) C. Adamo and V. Barone, J. Chem. Phys., 1998, 108, 664-675; (b) J. P. Perdew, K. Burke and Y. Wang, Phys. Rev. B: Condens. Matter, 1996, 54, 16533-16539.

12 A. M. Amado, S. M. Fiuza, M. P. M. Marques and L. A. E. Batista de Carvalho, J. Chem. Phys., 2007, 127, 185104.

13 (a) T. Visentin, E. Kochanski and A. Dedieu, THEOCHEM, 1998, 431, 255-265; (b) A. Milet and A. Dedieu, Theor. Chim. Acta, 1995, 92, 361-367.

14 S. D. Kirik, L. A. Solovyov, M. L. Blokhina, I. S. Yakimov and M. L. Blokhina, Acta Crystallogr., Sect. B: Struct. Sci., 1996, 52, 909-916.

15 J. F. Lopes, W. R. Rocha, H. F. Dos Santos and W. B. De Almeida, J. Chem. Phys., 2008, 128, 165103.

16 (a) R. S. Grev and H. F. Schaefer III, J. Chem. Phys., 1989, 91, 7305-7306; (b) L. Goodman and R. R. Sauers, J. Comput. Chem., 2007, 28, 269-275.

17 F. Grein, Theor. Chem. Acc., 2003, 109, 274-277.

18 C. W. Bauschlicher Jr., Chem. Phys. Lett., 1995, 246, 40-44.

19 A. D. Boese, J. Chem. Phys., 2003, 119, 3005-3014.

20 F. Jensen, Introduction to Computational Chemistry, John Wiley \& Sons, Sussex, England, 2003, p. 154.

21 Y. Tantirungrotechai, K. Phanasant, S. Roddecha, P. Surawatanawong, V. Sutthikhum and J. Limtrakul, THEOCHEM, 2006, 760, 189-192.

22 (a) A. P. Scott and L. Radom, J. Phys. Chem., 1996, 100, 16502-16513; (b) X. Zhou, C. J. M. Wheeless and R. Liu, Vib. Spectrosc., 1996, 12, 53-63; (c) X. Zhou, S. J. Mole and R. Liu, Vib. Spectrosc., 1996, 12, 73-79; (d) X. Zhou and R. Liu, Vib. Spectrosc., 1996, 12, 65-71; (e) E. F. C. Byrd, C. D. Sherrill and M. Head-Gordon, J. Phys. Chem. A, 2001, 105, 9736-9747; (f) B. Galabov, Y. Yamaguchi, R. B. Remington and H. F. Schaefer, J. Phys. Chem. A, 2002, 106, 819-832; (g) M. D. Halls, J. Velkovski and H. B. Schlegel, Theor. Chem. Acc., 2001, 105, 413-421.

23 S. M. Fiuza, A. M. Amado, M. P. M. Marques and L. A. E. Batista de Carvalho, J. Phys. Chem. A, 2008, 112, 3253-3259.

24 (a) D. Michalska and R. Wysokinski, Chem. Phys. Lett., 2005, 403, 211-217; (b) R. Wysokinski, K. Hernik, R. Szostak and D. Michalska, Chem. Phys., 2007, 333, 37-48.

25 H. F. Dos Santos, A. M. G. Do Val, A. C. Guimarães and W. B. De Almeida, Quim. Nova, 1999, 22, 732-736.

26 C. H. Perry, D. P. Athans and E. F. Young, Spectrochim. Acta, Part A, 1967, 23, 1137-1147.

27 (a) M. M. Nolasco, A. M. Amado and P. J. A. Ribeiro-Claro, ChemPhysChem, 2006, 7, 2150-2161; (b) M. Sardo, A. M. Amado and P. J. A. Ribeiro-Claro, J. Raman Spectrosc., 2009, 40, $1956-1965$. 